\title{
A internacionalização nas Geociências da USP: comparação entre coberturas da Web of Science e da Scopus no nível micro
}

\author{
Anderson de Santana \\ Mestre; Universidade de São Paulo, São Paulo, SP, Brasil \\ desantana.anderson@gmail.com \\ Rogério Mugnaini \\ Doutor; Universidade de São Paulo, São Paulo, SP, Brasil \\ mugnaini@usp.br
}

\begin{abstract}
Resumo: O estudo analisou a internacionalização da produção científica individual de pesquisadores do Instituto de Geociências da Universidade de São Paulo (IGc/USP) por meio de indicadores bibliométricos. Trata-se de um estudo de caso, com levantamento de dados e análise bibliométrica, e abordagem exploratória e descritiva. Foi definida uma amostra de 18 pesquisadores, cuja produção foi analisada segundo três categorias de indicadores: produção (nas fontes Dedalus/USP, Web of Science e Scopus), impacto e colaboração (somente nas bases internacionais). Com base nos resultados pôde-se constatar que a maioria dos pesquisadores da amostra aumentaram seu nível de publicação internacional. Além disso, sua produção se mostrou mais representativa na Scopus, quando comparada por meio de indicadores baseados em números absolutos de documentos indexados e citações recebidas. Contudo, ao se considerar indicadores normalizados, a vantagem da Scopus não se pronunciou na mesma magnitude, ainda que tenha sobressaído. Por fim, não se pode ignorar as especificidades das áreas, que se evidenciam nas práticas de comunicação científica, que inclui diversos fatores de escolha dos periódicos científicos, consequentemente implicando na internacionalização.
\end{abstract}

Palavras-chave: Internacionalização. Bibliometria. Geociências. Universidade de São Paulo. Performance Individual.

\section{Introdução}

O processo de comunicação dos resultados de pesquisa tem se constituído em uma das principais vias capazes de conferir capital científico. Entre as preocupações da comunidade científica, em especial nos países em desenvolvimento, está a necessidade de atingir patamares de internacionalização das instituições para atender os critérios de avaliação empreendidos por agências 
governamentais, bem como os estabelecidos pelas próprias instituições de ensino, que em geral tendem a embasar suas análises utilizando puramente parâmetros mensuráveis (quantitativos), incluindo os bibliométricos.

Rafols et al. (2016) explicam que países periféricos, na execução dos processos avaliativos da ciência que produzem, normalmente adotam métodos de avaliação quantitativa que são implementados com caráter normativo e rígido, mas por outro lado existem critérios implícitos que visam ao que os autores chamam de internacionalização da ciência, em detrimento da pesquisa de orientação local. Tal contexto, que no Brasil acaba se evidenciando de maneira explícita, em especial por conta de processos avaliativos como o da pós-graduação, acaba moldando o comportamento de parte dos pesquisadores.

Nesse sentido, a comunidade de especialistas em Bibliometria vem apresentando uma tendência crescente de analisar a produtividade e o impacto dos resultados de pesquisa em um nível mais granular, partindo-se do menor nível de agregação para o maior, criando-se métodos de análise no nível do pesquisador. Como exemplo, Abramo, Cicero e D’Angelo (2013) propuseram o indicador chamado de Fractional Scientific Strength, (em tradução livre: Força Científica Fracionária) que consiste em medir as citações normalizadas por campo de estudo das publicações de um pesquisador durante um período, ao mesmo tempo em que contabiliza a autoria de maneira fracionada. Já a proposta de Costas, Van Leeuwen e Bordons (2010) tem como objetivo desenvolver um esquema classificatório que permita (a) caracterizar e descrever diferentes perfis ou classes (níveis de carreira) de cientistas, com ênfase especial na identificação de "pesquisadores top" e (b) explorar diferentes aspectos do processo de pesquisa e se eles podem diferir entre cientistas de acordo com sua classe. Tal estudo permite avaliar a produtividade e impacto do pesquisador com relação à sua idade. Outro exemplo a ser citado é o da equipe de pesquisadores do Department for Bibliometrics and Publication Strategies da Biblioteca da Universidade de Viena, Áustria, que propõem uma série de critérios para o estabelecimento de relatórios bibliométricos individualizados, considerando as especificidades das áreas de conhecimento, entrevistas, além do uso de indicadores alternativos, dentre outros (GORRAIZ; WIELAND; GUMPENBERGER, 2016). 


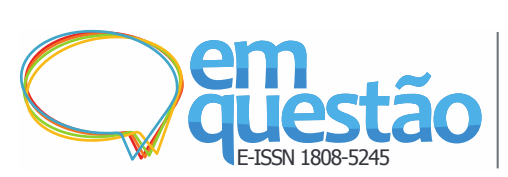

\section{A internacionalização nas Geociências da USP: comparação entre coberturas da Web of Science e da Scopus no nível micro \\ Anderson de Santana e Rogério Mugnaini}

A despeito do uso cada vez mais recorrente de indicadores desta natureza, muitos pesquisadores alertam para o uso exacerbado da bibliometria, na qual não deveria predominar a avaliação de resultados de pesquisa, tampouco o uso de um único indicador embasando decisões, devendo atentar para que questões de contexto, cultura e regionalismo das áreas sejam trabalhadas em conjunto (GLÄNZEL; DEBACKERE, 2003; RAFOLS et al., 2016). Contrariamente a essas recomendações, as avaliações no Brasil, em especial a da pós-graduação, conduzida pela Coordenação de Aperfeiçoamento de Pessoal de Nível Superior (CAPES), utiliza indicadores bibliométricos para nivelar os Programas de Pós-Graduação na avaliação do Sistema Nacional de PósGraduação (SNPG) (OLIVEIRA; AMARAL, 2017).

Ao mesmo tempo, o Brasil é um país que vem constituindo diversas fontes de informação próprias - como a Plataforma Lattes, o Projeto SciELO, Banco de Teses e Dissertações e os repositórios institucionais - e permitindo a realização de uma série de estudos que já vislumbram a concepção de indicadores mais condizentes com sua realidade. Mas mesmo havendo uma prática costumas de publicação em periódicos domésticos, os avanços rumo à internacionalização (LEITE; MUGNAINI; LETA, 2011; MUGNAINI; DIGIAMPETRI, 2015) - publicação em periódicos estrangeiros - é uma tendência presente, e claramente estimulada pela política científica nacional.

Diante desse cenário, propõe-se o uso de múltiplas fontes de informação, a fim de analisar o perfil de internacionalização da produção científica individual de pesquisadores da área de Geociências. Outro aspecto de importância central foi a comparação do desempenho dos pesquisadores, com base em indicadores bibliométricos das principais bases de dados internacionais - Web of Science (WoS) e Scopus. Tal constatação serviu ainda para verificar a hipótese de que pesquisadores com maior tendência de publicação em periódicos estrangeiros - ou aqueles cuja tendência de publicação nesses periódicos aumentou ao longo do período analisado - são os que apresentam indicadores de impacto mais expressivos, nas bases de dados internacionais.

Para tanto, a pesquisa se baseou no Instituto de Geociências da Universidade de São Paulo (IGc/USP), por ser o local no qual um dos pesquisadores realiza suas atividades profissionais. O IGc/USP foi criado em 
1969 e é considerado pioneiro no país na pesquisa de diversas áreas das Geociências. Possui três programas de pós-graduação e um corpo docente formado por 87 pesquisadores entre ativos, aposentados e sêniores.

Além disso, a USP atualmente se torna um exemplo oportuno para estudo de caso, pois apesar de haver iniciado seu processo de avaliação institucional em 1992 - que já contabiliza quatro ciclos de avaliação completos -, põe em marcha um novo modelo, com ciclos de cinco anos. Os docentes passarão a apresentar um projeto de pesquisa, que por sua vez deverá estar alinhado com seu Departamento e Unidade (OLIVEIRA; AGOPYAN, 2017). Tais elementos servirão tanto para a avaliação periódica, à qual o docente será submetido, quanto para a avaliação de progressão na carreira.

O presente estudo resulta de um aprofundamento de uma versão apresentada no $6^{\circ}$ Encontro Brasileiro de Bibliometria e Cientometria $\left(6^{\circ}\right.$ $\mathrm{EBBC}$ ), que naquele momento focava-se na análise da internacionalização, utilizando somente a Scopus como base internacional (SANTANA; MUGNAINI, 2018). Nesta versão estendida, indicadores similares da WoS foram adicionados, com vistas à análise comparativa de cobertura - não somente da produção, mas também do impacto.

\section{A internacionalização da produção científica}

A produção científica brasileira nos últimos 20 anos tem aumentado sua representatividade em bases como WoS e Scopus ${ }^{1}$, ocupando respectivamente as $15^{\mathrm{a}}$ e $16^{\mathrm{a}}$ posições dentre as nações com mais conteúdos indexados e as $16^{\mathrm{a}}$ e $20^{\mathrm{a}}$ dentre as com maior número de citações.

Os fatores que contribuíram para esse crescimento são variados, como o aumento da representatividade de periódicos brasileiros sendo indexados nas bases de dados (MUGNAINI; DIGIAMPETRI; MENA-CHALCO, 2014), e políticas públicas de incentivo à produtividade científica, como a concessão da bolsa de Produtividade em Pesquisa pelo Conselho Nacional de Desenvolvimento Científico e Tecnológico $(\mathrm{CNPq})^{2}$.

Também merecem destaque os esforços do Ministério da Educação do Brasil, com a avaliação do SNPG, conduzida pela Diretoria de Avaliação da 
CAPES. Ela pauta-se em padrões internacionalmente reconhecidos, com parâmetros que enfatizam a produção acadêmica dos professores, e estabelecendo uma escala de sete pontos como forma de ranqueamento dos Programas de Pós-Graduação (PPGs) - sendo reservados aos dois níveis mais altos aqueles qualificados como bons ou excelentes padrões internacionais de qualidade (BALBACHEVSKY, 2005).

A área de avaliação das Geociências define 20 critérios como condição para um PPG atingir as notas mais altas, dos quais destacam-se aqueles relacionados a produtividade e internacionalização:

[...] 2. as publicações dos docentes permanentes devem se dar dominantemente em periódicos internacionais dos estratos mais elevados do Qualis (A1, A2 e B1), livros e capítulos publicados no exterior em editoras qualificadas e livros nacionais de alta qualidade; [...] 7. projetos com financiamento internacional e participação em editais internacionais; [...] 8. produção intelectual em cooperação com pesquisadores estrangeiros; 13. participação qualificada (palestrante ou conferencista convidado) e apresentação de trabalhos em eventos científicos internacionais de alto nível acadêmico; [...] (CAPES, 2016, on-line).

No que tange à formação dos pós-graduandos, os critérios também se encontram relacionados à inserção desses em estágios internacionais (bolsas sanduíche), bem como o recebimento de alunos estrangeiros no PPG. Dessa forma, os critérios de avaliação e qualificação estabelecidos nos documentos de área têm delineado o perfil de publicação da ciência e, obviamente, os PPGs se planejam e reorganizam suas linhas de atuação com vistas a obter o necessário reconhecimento internacional, assim como viabilizar a nota do PPG, como ressalta Marrara (2007, p. 256):

A obtenção dessas notas, por sua vez, amplia o reconhecimento das IES e do programa bem avaliado, além de lhes permitir o acesso a certos recursos financeiros, tais como os provenientes do Programa de Excelência Acadêmica (PROEX), exclusivos para programas com reconhecido nível de excelência.

É possível verificar nos referidos documentos, semelhanças e diferenças nas visões e proposições das diversas áreas sobre como pode se dar a internacionalização das atividades acadêmicas. Contudo, apesar da literatura especializada revelar a complexidade para mensuração do fenômeno em questão, entre os indicadores bibliométricos utilizados pelas áreas na avaliação 
dos PPGs, no que tange à classificação de periódicos (sistema Qualis) no ciclo avaliativo de 2013-2016: o Fator de Impacto do Journal Citation Reports ${ }^{3}$ (JCR) era utilizado por $81 \%$ das áreas; o SCImago Journal Ranking ${ }^{4}$ (SJR), por 56\%; já outros indicadores de impacto, como o índice h e citações por documento, foram utilizados por um número menor de áreas; e apenas sete áreas, das 49, não mencionaram utilizar indicador bibliométrico (OLIVEIRA; AMARAL, 2017).

Zitt e Bassecoulard (1998) comentam que vários autores já apresentaram critérios específicos para análise do nível de internacionalidade de um periódico científico, dos quais destacam: a distribuição nacional dos autores (quantidade de autores do país de publicação do periódico); distribuição nacional dos usuários (incluindo leitores, assinantes e citadores); vinculação dos coautores (proporção de coautorias nacionais e internacionais); idioma e publicação por editoras comerciais; e nacionalidade dos conselhos editoriais. Já Buela-Casal et al. (2006) propõem um conjunto mais diversificado de critérios para o desenvolvimento de um índice de internacionalidade de periódicos baseado no idioma da publicação, no país de publicação, na inclusão do termo “internacional" no título, na inclusão em bases de dados internacionais, no Fator de Impacto, na afiliação a uma instituição ou associação internacional, na distribuição multinacional dos membros do conselho editorial, na distribuição multinacional dos editores associados, na distribuição multinacional de autores, na distribuição multinacional de usuários, nos padrões de colaboração internacional, e no acesso on-line.

Como se pode ver, pouco tem sido absorvido do que a comunidade de especialistas em estudos bibliométricos tem apresentado, desde a implantação do Qualis em 1998. Os índices de citação de empresas comerciais são as principais fontes utilizadas, sendo portanto necessário avaliar os cenários delineados pelos indicadores oferecidos pelas mesmas. Por essa razão, a abordagem bibliométrica em nível micro, a partir de uma análise multidimensional da produção científica dos pesquisadores do IGc/USP permitiu a observação da heterogeneidade entre os perfis de pesquisadores de PPGs similares, o que normalmente não pode ser observado quando da avaliação de dados agregados, no nível do PPG, conforme realizado pela CAPES. 


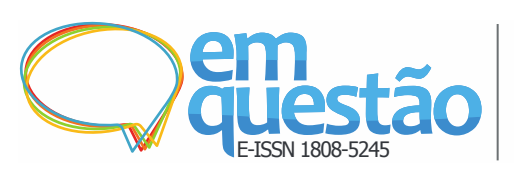

\section{Metodologia}

Foi realizado um estudo de caso, com abordagem exploratória e descritiva, utilizando-se de levantamento de dados e análise bibliométrica. Uma amostra de 18 pesquisadores do IGc/USP foi tomada de entre aqueles habilitados em algum de seus três PPGs, selecionados a partir de critérios descritos mais a diante.

As bases internacionais utilizadas, WoS e Scopus, foram utilizadas, havendo sido pertinente contar com a possibilidade de identificação dos pesquisadores, eliminando assim possíveis ambiguidades relativas à homonímia.

No intuito de garantir dados robustos, e ao mesmo tempo consistência das tendências a analisar, a amostra de pesquisadores foi especificada buscando selecionar aqueles que apresentassem uma quantidade mínima de publicações (número de artigos, independente de base de dados específica), além de atuarem ativamente em algum dos PPGs do IGc. De um total de 85 pesquisadores, dos quais, um total de 47 estavam credenciados em pelo menos dois ciclos avaliativos da Capes (considerando os quatro triênios compreendidos no período de 2001 a 2012, assim como o quadriênio de 2013 a 2016). Então, dois critérios foram aplicados em relação à produtividade dos mesmos, considerando o número de artigos em periódicos registrados no Dedalus. O primeiro exigiu pelo menos 25 artigos no período de 2001-2016 e o segundo pelo menos dez artigos em cada um dos dois subperíodos determinados, quais sejam: 2001-2009 (T1T3) e 2010-2016 (T4 e Q1). A aplicação desses dois critérios determinou uma amostra de 18 pesquisadores.

Para viabilizar a análise comparativa de desempenho dos mesmos, com base em indicadores de impacto e colaboração de sua produção - somente artigos -, foram utilizadas as ferramentas ${ }^{5}$ InCites (que utiliza dados da WoS) e SciVal (da Scopus). Para analisar a cobertura dessas bases procedeu-se comparação com o Dedalus, por ser uma fonte completa da produção dos pesquisadores. Os indicadores utilizados foram divididos em três subgrupos, conforme apresentado na Figura 1, na qual pode-se observar quais indicadores são comuns ou específicos de cada base. Tais indicadores, foram distribuídos e somados, de acordo com a data de publicação dos artigos, nos cinco ciclos de 


\section{A internacionalização nas Geociências da USP: comparação entre coberturas da Web of Science e da Scopus no nível micro \\ Anderson de Santana e Rogério Mugnaini}

avaliação (T1-Q1) e no caso do FWCI e CNCI foram utilizadas as médias para o período.

A fim de agrupar os pesquisadores de acordo com o perfil delineado pelo conjunto completo de indicadores supracitados, calculados com base no período completo, foi utilizada a Análise Multivariada, que possibilitou a determinação de clusters, com base na distância euclidiana, utilizando o software Minitab.

Figura 1 - Indicadores obtidos das bases internacionais

\begin{tabular}{|c|c|c|c|}
\hline \multirow[t]{2}{*}{ Indicadores } & \multirow[t]{2}{*}{ Abreviações } & \multicolumn{2}{|c|}{ Bases } \\
\hline & & WoS & Scopus \\
\hline \multicolumn{4}{|l|}{ Produção (somente estes consideram o Dedalus) } \\
\hline Total de artigos registrados no Dedalus & Doc. Dedalus & & \\
\hline $\begin{array}{l}\text { Total e percentual de artigos indexados WoS e } \\
\text { Scopus em relação ao total de artigos no } \\
\text { Dedalus }\end{array}$ & $\begin{array}{l}\text { \% Doc. WoS / } \\
\text { Scopus }\end{array}$ & & \\
\hline $\begin{array}{l}\text { Percentual de artigos no } 1^{\circ} \text { quartil do JCR } \\
\text { (WoS) e SJR (Scopus) em relação ao total de } \\
\text { artigos indexados nas bases }\end{array}$ & $\begin{array}{l}\text { \% Doc. Q1 JCR / } \\
\text { SJR }\end{array}$ & & \\
\hline \multicolumn{4}{|l|}{ Impacto } \\
\hline Total de citações recebidas & Cit. WoS / Scopus & & \\
\hline Citações por artigo & $\begin{array}{l}\text { Cits./Doc. WoS / } \\
\text { Scopus }\end{array}$ & & \\
\hline Percentual de artigos citados & $\begin{array}{l}\text { \% Doc. Cit. WoS / } \\
\text { Scopus }\end{array}$ & & \\
\hline $\begin{array}{l}\text { Impacto de Citação Normalizado pela } \\
\text { Categoria (Category Normalized Citation Impact } \\
\text { - CNCI) }\end{array}$ & WoS CNCI & & \\
\hline $\begin{array}{l}\text { Impacto de Citação Ponderada no Campo } \\
\text { (Field-Weighted Citation Impact - FWCI - para } \\
\text { a Scopus) }\end{array}$ & Scopus FWCI & & \\
\hline \multicolumn{4}{|l|}{ Colaboração } \\
\hline Percentual de colaboração internacional & $\begin{array}{l}\text { \% Col. Int. WoS / } \\
\text { Scopus }\end{array}$ & & \\
\hline
\end{tabular}

Fonte: Elaborado pelos autores (2018).

Além das bases de dados internacionais, outras fontes de dados corporativas foram utilizadas, colhendo do já mencionado banco de dados Dedalus o registro de toda a produção intelectual dos pesquisadores da amostra. Outras fontes foram essenciais para levantamento de informações sobre os pesquisadores e sua carreira acadêmica, resultando em um banco de dados relacional - no Microsoft Access (Figura 2). 
A partir do Dedalus, pôde-se descrever o perfil de publicação em periódicos domésticos dos pesquisadores, segundo o indicador proposto por Leite, Mugnaini e Leta (2011): se o pesquisador publicou até $20 \%$ em periódicos domésticos, seu nível de publicação foi considerado [1]; caso contrário, se publicou até 40\%, [2]; e assim por diante, até o nível de maior percentual de publicações nacionais, cujo percentual somaria mais do que $80 \%$, e, portanto, nível [5]. Determinou-se esse nível para cada um dos subperíodos permitindo compará-los com o objetivo de verificar se houve diminuição do nível de publicação nacional - ou seja, se ocorreu internacionalização.

Figura 2 - Fluxograma de desenvolvimento da Base de Dados da Produção Intelectual do $\mathrm{IGc/USP}$

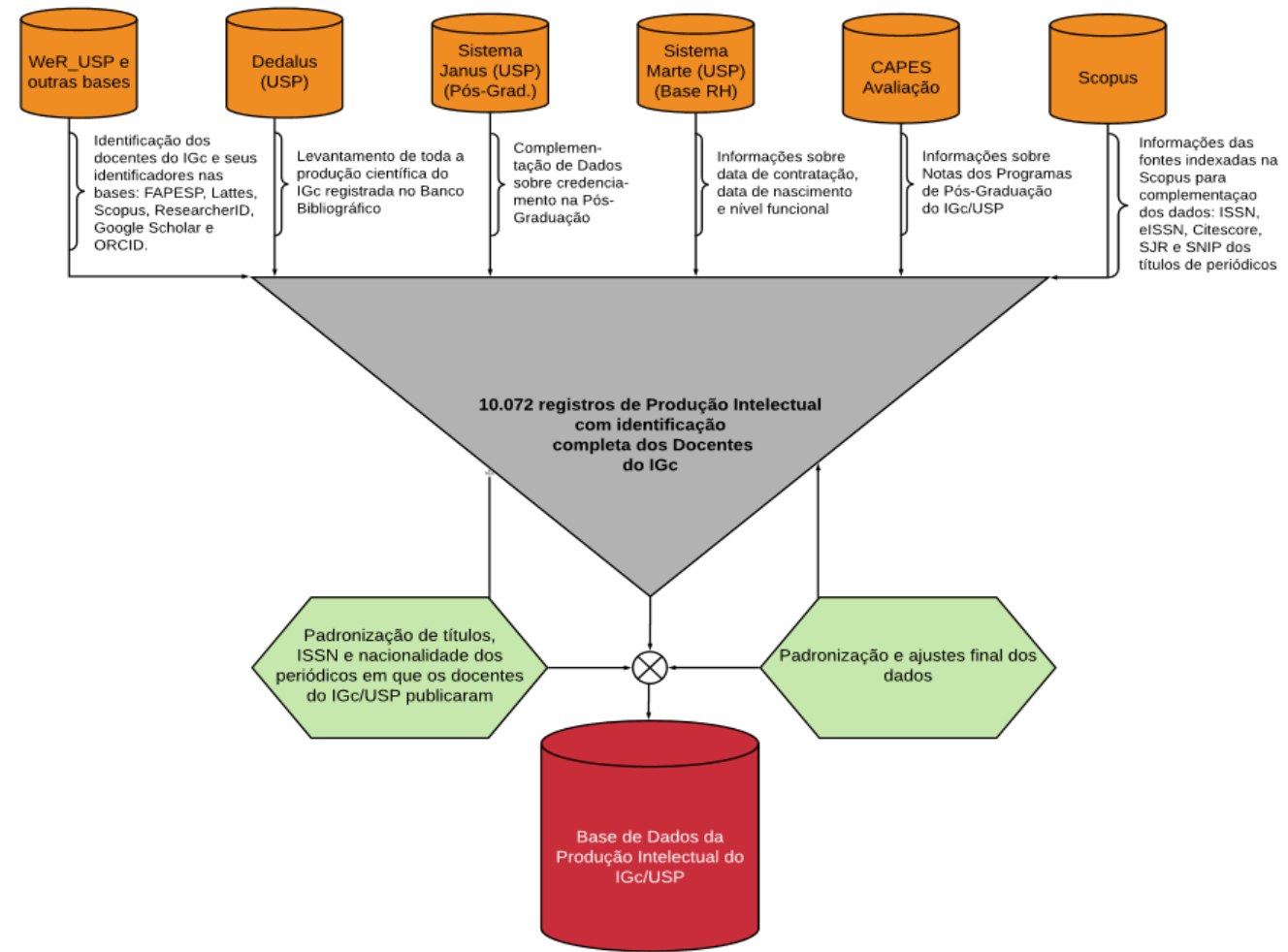

Fonte: Elaborado pelos autores (2018).

Tais procedimentos visaram diferenciar os pesquisadores segundo os seguintes perfis: que apresentam diferentes níveis de produção (extremo doméstico e extremo internacional) ou aqueles que apresentaram significativa mudança de nível entre os subperíodos. Assim estes perfis puderam ser utilizados para comparação com o perfil delineado pelos indicadores de desempenho apresentados na Figura 1. 


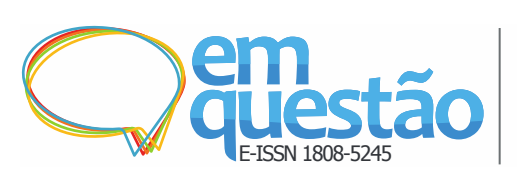

\section{Resultados}

A análise do desempenho dos pesquisadores da amostra, baseada nos indicadores bibliométricos relativos à sua produção científica é apresentada na Figura 3. Nesta etapa optou-se por considerar o período completo (2001-2016), sem considerar evoluções temporais. Em cada gráfico da Figura 3, indicadores similares são comparados, para possibilitar a comparação do desempenho de cada um dos 18 pesquisadores (enumerados no sentido horário), segundo o contexto proporcionado pela WoS e Scopus. Um eixo radial, do centro para a extremidade superior, apresenta o percentual atingido no indicador de cada base, em cada um dos gráficos. A única exceção é a Figura 3D, que diferente das demais, apresenta (além das linhas azul e laranja) duas áreas, que dizem respeito aos indicadores de impacto normalizado de cada base (cinza corresponde ao CNCI da WoS e amarela ao FWCI da Scopus) - cuja unidade de medida não é percentual e por essa razão os números absolutos são apresentados na respectiva cor, no vértice correspondente a cada pesquisador.

Pode-se constatar, por meio da Figura 3A, que a produção dos pesquisadores se mostra mais pronunciada, quando considerada a base Scopus. Tal discrepância se deve ao fato de nem todos os artigos registrados no Dedalus haverem sido publicados em periódicos indexados nessas bases internacionais. Tais bases possuem critérios de seleção específicos, resultando num percentual de abrangência díspar, quando considerada cada uma. Tomando como exemplo o pesquisador 7 , constatamos que $100 \%$ de sua produção é considerada na Scopus, ao passo que a base WoS abarca apenas cerca de $73 \%$ de seus artigos. Já no caso dos pesquisadores 1, 2, 4, 8, 13 e 15, esse percentual é bastante similar, ainda que sempre maior na Scopus.

Na Figura 3B observa-se o percentual dos artigos que foram publicados nos periódicos de maior impacto ( $1^{\circ}$ quartil do JCR e SJR), dentre a coleção de periódicos de cada base. O pesquisador 4, cujo percentual é bem próximo de $80 \%$ nas duas bases, permite a observação de que selecionou os periódicos de maior impacto para publicação da maior parte de seus artigos em periódicos indexados (haja vista que na Figura 3A seu percentual de publicação nas bases fica entre 55 e 62\%). Já os pesquisadores 1, 2, 9, 10, 11, 13 e 15 publicam num 
percentual maior de periódicos de maior impacto da Scopus, enquanto que apenas o pesquisador 16 revela um perfil oposto publicando em periódicos de mais alto Fator de Impacto (indicador do JCR, relacionado à WoS). Pode ser natural que o maior percentual de artigos em periódicos do $1^{\circ}$ quartil do SJR seja mais prevalente, dado o fato de que a área de avaliação das Geociências na CAPES considere este indicador - para classificação dos periódicos nos diferentes estratos - em detrimento do Fator Impacto. É de se esperar, portanto, que os pesquisadores tenham o SJR como parâmetro de escolha dos periódicos para submissão de seus manuscritos.

Notamos que em ambas as figuras, a Scopus se revela uma base mais abrangente para representação da produção dos pesquisadores, e ainda, os artigos da maioria deles foram publicados em periódicos de maior impacto, tendendo a oferecer maior visibilidade. Contudo, ao se observar o percentual de artigos citados (Figura 3C), a discrepância entre as fontes é menos pronunciada, havendo a mesma quantidade de pesquisadores, cujo percentual é maior: na WoS os pesquisadores 6, 7, 10, 11 e 18; e na Scopus, 2 9, 12, 13 e 14. Destacase que no caso dos pesquisadores 10, 11 e 18, o percentual de artigos citados da WoS é significativamente mais pronunciado que na Scopus.

Concluímos, portanto, que quando se trata de produtividade (não importa se em periódicos de mais alto impacto), a base Scopus mostra-se mais representativa, enquanto que, em se tratando do impacto baseado no percentual de artigos citados, os contextos são muito similares, com leve tendência a favor da WoS. Para se ter uma visão mais equitativa do cenário, a utilização de indicador de impacto normalizado, de cada uma das bases permite observar (áreas da Figura 3D) um comportamento muito similar, com algumas exceções: o pesquisador 16 apresenta impacto normalizado significativamente maior na WoS, mesmo que o seu percentual de artigos citados não apresentou diferença entre as bases (Figura 3C); pesquisadores 3, 11, 12 e 18 também apresentaram impacto normalizado ligeiramente maior na $\mathrm{WoS}$; o destaque de impacto normalizado na Scopus é evidenciado pelo pesquisador 13 (que na Figura 3B sinaliza alto percentual de artigos no $1^{\circ}$ quartil do SJR), e unem-se a ele os pesquisadores 2 e 14 . 


\section{A internacionalização nas Geociências da USP: comparação entre coberturas da Web of Science e da Scopus no nível micro \\ Anderson de Santana e Rogério Mugnaini}

Vale observar ainda que o percentual de artigos publicados com coautor(es) estrangeiro(s) (linhas da Figura 3D) é bastante similar entre as bases, mas ligeiramente maior na WoS (linha azul), com exceção dos pesquisadores 4, 8, 13 e 14. Outro aspecto que merece atenção é a correlação entre as áreas (impacto normalizado) e linhas (colaboração internacional), que é percebida pela similaridade das formas das figuras, com exceção dos pesquisadores 16 e 17, para os quais o impacto normalizado é mais acentuado; e o pesquisador 6, cujo percentual de colaboração internacional é que destoa.

Figura 3 - Performance comparativa dos 18 pesquisadores na Scopus e WoS, segundo percentual de documentos indexados

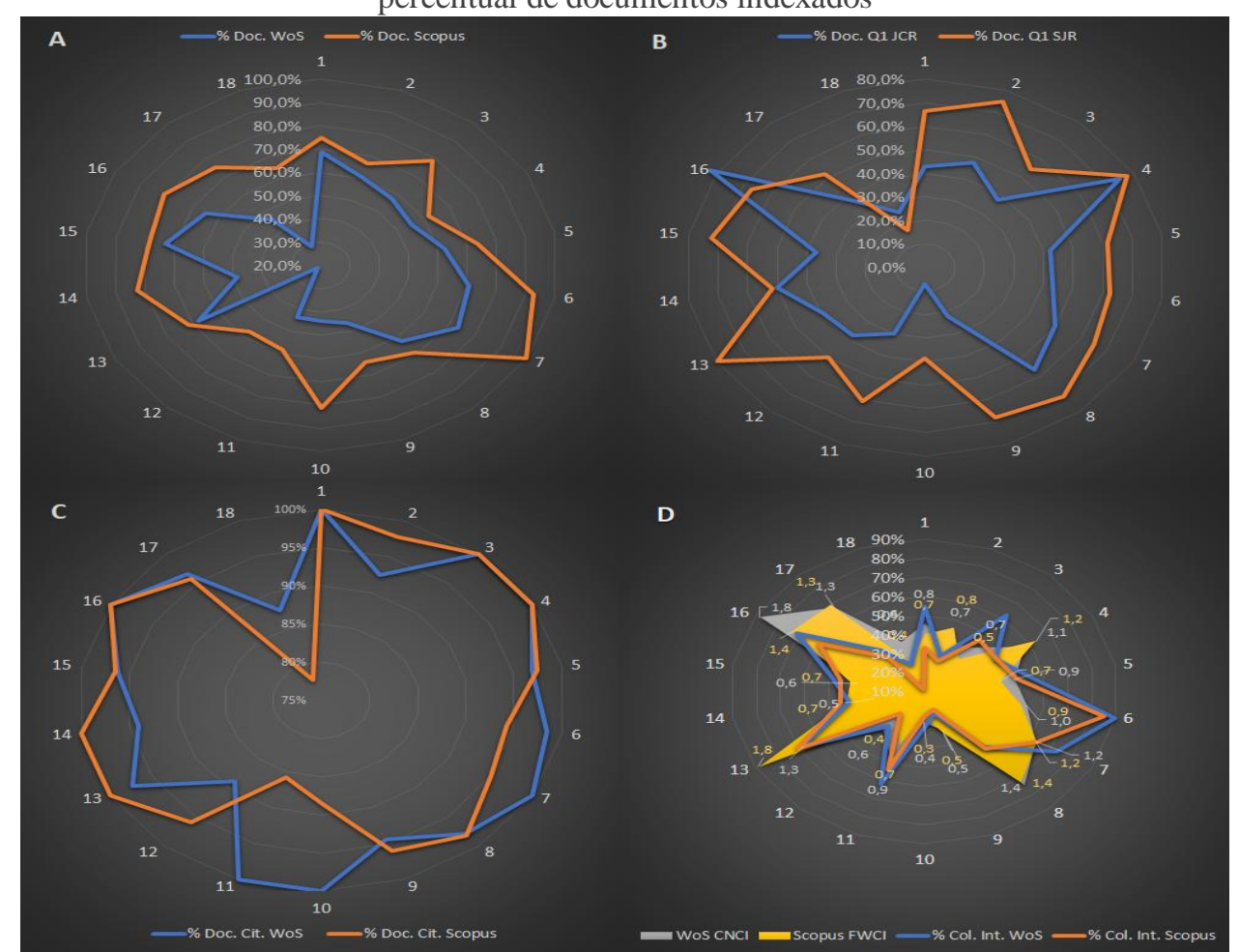

Legenda: (a), percentual de documentos no $1^{\circ}$. Quartil do SJR e JCR; (b) percentual de documentos citados; (c), FWCI, CNCI e percentual de colaboração internacional; (d) período 2001-2016.

Fonte: Dedalus/USP, Incites/WoS e SciVal/Scopus.

No intuito de agrupar os pesquisadores segundo os valores dos indicadores de sua produção que compartilham características comuns, recorreuse à Análise Estatística Multivariada, que a partir da distância euclidiana entre os valores, permitiu a definição de cinco clusters. Os clusters podem ser diferenciados segundo os tons de vermelho nas células das colunas da Tabela 1, 


\section{A internacionalização nas Geociências da USP: comparação entre coberturas da Web of Science e da Scopus no nível micro \\ Anderson de Santana e Rogério Mugnaini}

evidenciando quais indicadores (média dos integrantes de cada cluster) caracterizam o desempenho dos pesquisadores, justificando o agrupamento.

De modo geral, pode-se perceber na Tabela 1 que os indicadores da Scopus são mais pronunciados que os da WoS, com algumas exceções observadas, como nas médias dos pesquisadores do Cluster 4, que apresentou os menores valores em geral. Outra exceção se dá no percentual de colaborações internacionais, que para todos os clusters se apresentou significativamente maior na WoS.

Primeiramente, podemos observar que o Cluster 3 evidencia valores mais pronunciados em quase a totalidade dos indicadores da WoS (com exceção da média de citações por documento), e em relação aos indicadores da Scopus se destacam pelo maior percentual de sua produção naquela base, além do indicador de impacto normalizado (FWCI), percentual de artigos em periódicos de alto impacto (Q1 SJR) e percentual de colaboração internacional.

Já o Cluster 5 é o único formado por apenas um pesquisador, o de número 17 , cujo destaque se dá principalmente nos indicadores de impacto da Scopus, e apresentando média de pouco mais que 32 citações por artigo na WoS.

Tabela 1 - Perfil dos clusters resultantes da Análise Multivariada, de acordo com as médias de desempenho dos pesquisadores de cada cluster, em cada indicador - período 2001-2016

\begin{tabular}{|c|c|c|c|c|c|}
\hline \multirow{2}{*}{ Indicador } & \multicolumn{5}{|c|}{ Cluster } \\
\hline & 1 & 2 & 3 & 4 & 5 \\
\hline$\%$ Doc. WoS & $56,2 \%$ & $59,3 \%$ & $68,0 \%$ & $31,3 \%$ & $45,0 \%$ \\
\hline Cit. WoS & 221,167 & 377 & 1073,8 & 112 & 935 \\
\hline Cits./Doc. WoS & 11,072 & 15,037 & 27,43 & 11,607 & 32,24 \\
\hline$\%$ Docs. Cit. WoS & $97,5 \%$ & $96,5 \%$ & $99,0 \%$ & $92,1 \%$ & $97,0 \%$ \\
\hline WoS CNCI & 0,655 & 0,857 & 1,33 & 0,53 & 1,3 \\
\hline$\%$ Doc. Q1 JCR & $36,6 \%$ & $54,9 \%$ & $54,0 \%$ & $23,2 \%$ & $37,0 \%$ \\
\hline$\%$ Col. Int. WoS & $48,4 \%$ & $42,8 \%$ & $70,0 \%$ & $29,0 \%$ & $38,0 \%$ \\
\hline$\%$ Doc. Scopus & $72,9 \%$ & $67,4 \%$ & $83,0 \%$ & $67,6 \%$ & $75,0 \%$ \\
\hline Cit. Scopus & 349,5 & 496 & 1329,2 & 182,667 & 1516 \\
\hline Cits./Doc. Scopus & 13,361 & 16,951 & 27,76 & 8,283 & 31,58 \\
\hline \% Docs. Cit. Scopus & $96,4 \%$ & $98,4 \%$ & $97,0 \%$ & $87,4 \%$ & $96,0 \%$ \\
\hline Scopus FWCI & 0,633 & 0,903 & 1,34 & 0,343 & 1,34 \\
\hline \% Doc. Q1 SJR & $62,3 \%$ & $71,4 \%$ & $69,0 \%$ & $35,0 \%$ & $52,0 \%$ \\
\hline$\%$ Col. Int. Scopus & $40,3 \%$ & $40,1 \%$ & $66,0 \%$ & $19,7 \%$ & $33,0 \%$ \\
\hline
\end{tabular}

Fonte: Dedalus/USP, Incites/WoS e SciVal/Scopus. 
Ainda na Tabela 1, outro cluster cujos pesquisadores se destacaram em diversos indicadores foi o Cluster 2, sendo que quase todos os artigos desse grupo são citados (em ambas as bases ultrapassa os 96\%), e selecionam os periódicos em que publicam (54,9\% dos artigos no Q1 do JCR e 71,4\% no Q1 do SJR).

Os pesquisadores do Cluster 1 (Tabela 1) apresentam desempenho muito similar ao do Cluster 2, em relação aos indicadores, destoando pelo menor impacto na WoS (citações por artigo e impacto normalizado CNCI), assim como na Scopus (ainda que com menor discrepância), e pelo maior percentual de artigos em periódicos indexados na Scopus (72,9\%).

E, por fim, conforme mencionado anteriormente, os pesquisadores do Cluster 4 foram os que apresentaram menor desempenho na totalidade dos indicadores, revelando menos de um terço de seus artigos em periódicos indexados na WoS, e ainda, menos de um quarto desses no Q1 do JCR. Apesar da Scopus abarcar dois terços da produção dos pesquisadores, as citações não são expressivas. Por outro lado, o percentual de artigos que receberam alguma citação em ambas as bases não é desprezível, mas pelo jeito não acumulou um número expressivo, haja vista o baixo percentual de colaboração internacional.

Havendo delineado o perfil dos pesquisadores, de acordo com o desempenho em indicadores bibliométricos, conforme observado nos diferentes clusters, a Tabela 2 permite observar os integrantes de cada cluster, com respectivo perfil de publicação em periódicos domésticos e transição rumo à internacionalização entre os subperíodos.

Pode-se notar na Tabela 2 que os pesquisadores do Cluster 3 são os que apresentam menor nível de publicação em periódicos domésticos (1 ou 2) no segundo subperíodo (T4-Q1). Os três primeiros (pesquisadores 6, 7 e 8) subiram um nível rumo à internacionalização, ou seja, diminuíram seu percentual de artigos em periódicos domésticos do subperíodo T1-T3 para T4-Q1. Os demais apresentaram comportamento diferente: o pesquisador 16 transitou no sentido contrário, tendo o percentual de artigos em periódicos nacionais aumentados entre os subperíodos; e o pesquisador 13 se manteve no nível 2, de um triênio para o outro, não havendo transitado. O Cluster 3 é o único com predominância de professores sênior, cujo desempenho nos indicadores bibliométricos nas 
bases internacionais (Tabela 1) foi o mais pronunciado, sinalizando correlação entre inserção internacional da produção científica e desempenho nos indicadores de impacto.

Já o Cluster 5, que corresponde ao pesquisador 17, avançou um nível, no rumo contrário à internacionalização (Tabela 2). Chama a atenção o seu maior percentual de seus artigos na Scopus (75\%) em relação à WoS (45\%), que se deve ao fato de publicar parte significativa de seus artigos em periódicos brasileiros que estão indexados apenas na WoS. Como visto na Tabela 1, o seu desempenho é mais pronunciado na Scopus.

O Cluster 2, que congrega os pesquisadores 2, 4 e 5, é o segundo mais internacionalizado, sendo os que mais transitaram rumo à internacionalização (o pesquisador 2 foi do nível 5 para o nível 2, entre os subperíodos), conforme a Tabela 2. Mais uma evidência a favor da hipótese desta pesquisa, pois esse cluster se destacou nos indicadores bibliométricos pelo alto percentual de artigos citados e percentual de artigos em periódicos do $1^{\circ}$ quartil, em ambas as bases (Tabela 1).

O Cluster 1, por sua vez, é o mais numeroso, e mais heterogêneo no que diz respeito à transição rumo à internacionalização (Tabela 2): os pesquisadores 1, 3, 9 e 11 avançaram respectivamente 3, 2, 1 e 1 níveis; o pesquisador 14 ficou estagnado no nível 4 (entre 60 e $80 \%$ da produção em periódicos nacionais); e o pesquisador 15 regrediu um nível. No que diz respeito ao desempenho nas bases internacionais (Tabela 1), os indicadores desse cluster não se destacaram pelo volume de citações, tendo destaque de impacto apenas no percentual de artigos citados em ambas as bases.

E finalmente no Cluster 4 estão os pesquisadores com perfil menos internacionalizado, haja vista os seus perfis de publicação em periódicos domésticos mais pronunciados, em ambos os subperíodos, com transição positiva rumo à internacionalização apenas para os pesquisadores 10 e 12 (Tabela 2). O cenário apresentado para esse grupo de pesquisadores está em consonância com o perfil menos pronunciado do desempenho em indicadores bibliométricos, conforme observado na Tabela 1, e consequentemente, também alinha-se com a hipótese de pesquisa. 
Anderson de Santana e Rogério Mugnaini

Tabela 2 - Pesquisadores integrantes dos clusters, função, perfil de publicação em periódicos domésticos (subperíodos T1-T3 e T4-Q1) e transição rumo à internacionalização

\begin{tabular}{|c|c|c|c|c|c|}
\hline \multirow{2}{*}{ Pesquisador } & \multirow{2}{*}{ Função } & \multirow{2}{*}{ Cluster } & \multicolumn{2}{|c|}{ Perfil de publicação em periódicos domésticos } & \multirow{2}{*}{$\begin{array}{c}\text { Transição à } \\
\text { internacionalização }\end{array}$} \\
\hline & & & T1-T3 & T4-Q1 & \\
\hline 1 & Prof. Ativo & \multirow{6}{*}{1} & 4 & 1 & 3 \\
\hline 3 & Prof. Ativo & & 4 & 2 & 2 \\
\hline 9 & Prof. Ativo & & 3 & 2 & 1 \\
\hline 11 & Prof. Ativo & & 4 & 3 & 1 \\
\hline 14 & Prof. Sênior & & 4 & 4 & 0 \\
\hline 15 & Prof. Ativo & & 1 & 2 & -1 \\
\hline 2 & Prof. Ativo & \multirow{3}{*}{2} & 5 & 2 & 3 \\
\hline 4 & Prof. Sênior & & 4 & 2 & 2 \\
\hline 5 & Prof. Ativo & & 4 & 2 & 2 \\
\hline 6 & Prof. Ativo & \multirow{5}{*}{3} & 2 & 1 & 1 \\
\hline 7 & Prof. Sênior & & 2 & 1 & 1 \\
\hline 8 & Prof. Sênior & & 3 & 2 & 1 \\
\hline 13 & Prof. Sênior & & 2 & 2 & 0 \\
\hline 16 & Prof. Ativo & & 1 & 2 & -1 \\
\hline 10 & Prof. Sênior & \multirow{3}{*}{4} & 4 & 3 & 1 \\
\hline 12 & Prof. Ativo & & 5 & 4 & 1 \\
\hline 18 & Prof. Ativo & & 4 & 5 & -1 \\
\hline 17 & Prof. Sênior & 5 & 2 & 3 & -1 \\
\hline
\end{tabular}

Legenda: Nível de publicação nacional é determinado a partir do percentual de artigos em periódicos nacionais, sendo: [1] 0 |---| 20\%; [2] 20 ---| 40\%; [3] 40 ---| 60\%; [4] 60 ---| 80\%; [5] $80--\mid 100 \%$.

Fonte: Dedalus/USP, Incites/WoS e SciVal/Scopus.

A fim de se obter um panorama das diferenças entre a cobertura das bases nos indicadores, no nível individual, assim como o efeito cumulativo de sua evolução ao longo dos cinco ciclos avaliativos, a Tabela 3 apresenta a diferença percentual acumulada da Scopus, em relação à WoS - o que busca mensurar a vantagem da Scopus.

Neste comparativo, podemos notar que em números absolutos, todos os pesquisadores têm um saldo positivo percentual ao longo dos períodos, sinalizando que o montante de artigos e citações na Scopus é melhor representado. No entanto, em se tratando dos indicadores de impacto normalizado, a prevalência não é exclusiva da Scopus, ainda que o percentual mais pronunciado seja do pesquisador 14 , com aumento acumulado de $234 \%$, seguido pelos pesquisadores 13, 1 e 9 (Tabela 3). Observamos que três deles estão no Cluster 1, que conforme mostra a Tabela 1 não se destacam por esse indicador, o que pode decorrer do fato do pesquisador 11 apresentar o maior decréscimo acumulado (-224\%, que representa a desvantagem da Scopus, no caso deste pesquisador), contrabalanceando a média do grupo. 


\section{A internacionalização nas Geociências da USP: comparação entre coberturas da Web of Science e da Scopus no nível micro \\ Anderson de Santana e Rogério Mugnaini}

Tabela 3 - Soma da diferença percentual de cada indicador (total de artigos, total de citações e impacto normalizado) da Scopus, em relação à WoS, ao longo dos cinco ciclos avaliativos (T1 a

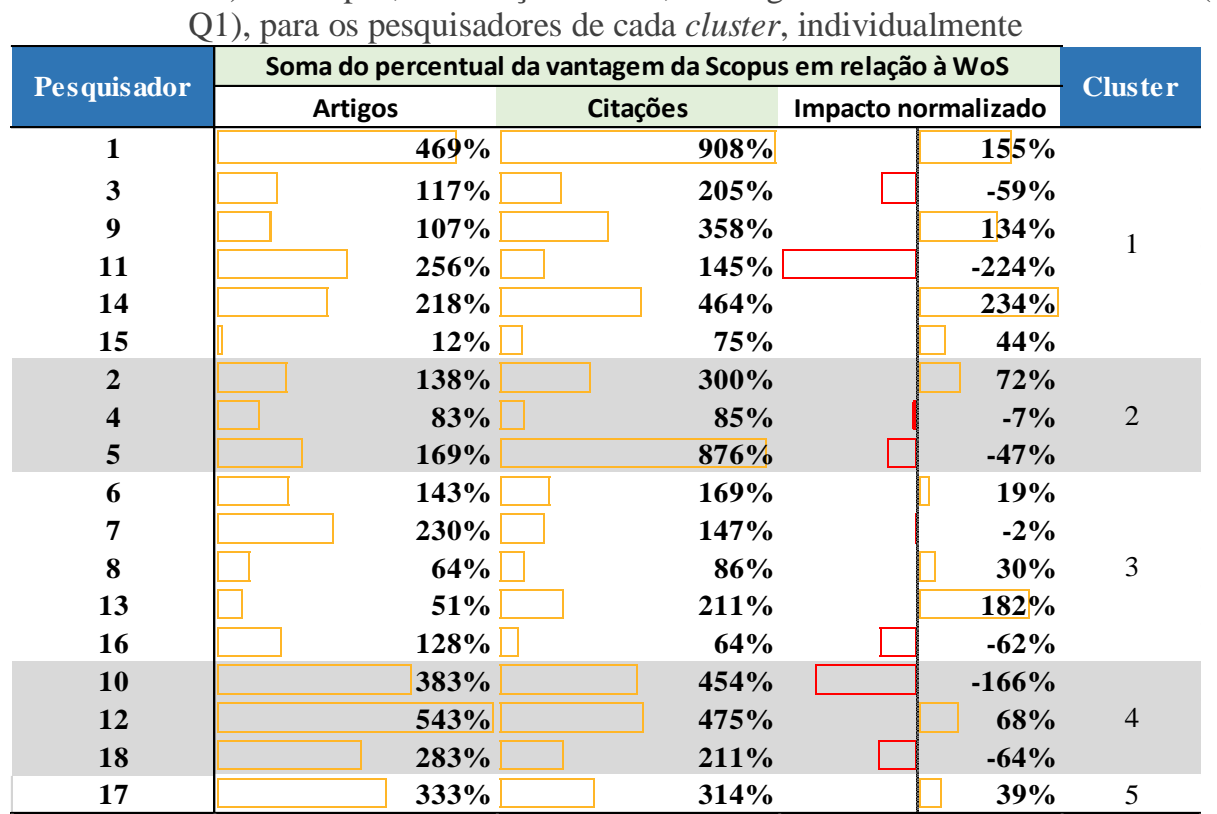

Legenda: A diferença percentual foi calculada para cada um dos indicadores, em cada um dos 5 ciclios avaliativos. A fórmula [( $\left.\left.\mathrm{Art}_{\text {Scopus }}-\mathrm{Art}_{W o S}\right) / \mathrm{Art}_{W o S}\right]$ exemplifica o cálculo que mensura a vantagem da Scopus em relação à WoS, para o indicador total de artigos. Após calcular para cada ciclo, somou-se o resultado dos 5 ciclos, sendo este o percentual apresentado nas células da tabela.

Fonte: Incites/WoS e SciVal/Scopus.

Os pesquisadores 10 e 11 são os que apresentam maior evidência de evolução de impacto na WoS (Tabela 3), o que coaduna com o alto percentual de documentos dos mesmos, que receberam citações na WoS, significativamente maior que o percentual da Scopus, conforme observado na Figura 3C, denotando que a captação de citações por todos os artigos na WoS pode influenciar o impacto obtido. No mesmo Cluster (4) do pesquisador 10, o de número 18 também apresenta impacto normalizado superior na WoS.

Os demais pesquisadores apresentaram um impacto normalizado maior na Scopus, sugerindo haver tendência à vantagem dessa fonte, considerando a produção científica desta amostra de pesquisadores das Geociências da USP.

\section{Considerações finais}

Os resultados da pesquisa, apesar de se pautarem em um perfil individual de 18 pesquisadores, devem ser analisados em conjunto, uma vez que não se pretendeu comparar os desempenhos entre eles. Antes de se pretender definir a base ideal para mensuração de performance, é preciso compreender os efeitos da cobertura 
nas diferentes dimensões que os indicadores bibliométricos utilizados buscaram refletir.

Exercícios avaliativos devem se basear em análises comparativas de cobertura entre diferentes bases de dados, para que não ignore informação importante. Como se pôde observar neste estudo, a Scopus prevaleceu à WoS na captação do impacto das publicações dos pesquisadores. Mesmo que em nível micro, esses resultados apontam para a necessidade de análise prévia para a adoção de fontes de informação. Contudo, não se pode ignorar as especificidades das áreas, que se evidenciam nas práticas de comunicação científica, ou seja, o que se observou nas Geociências poderá não valer para outras áreas.

Outro aspecto de importância central para o estudo se deu na análise da relação entre duas vias de internacionalização da produção científica. Os resultados apontaram para a confirmação da hipótese de que os pesquisadores com maior percentual de artigos em periódicos estrangeiros - ou cujo percentual vem aumentando entre os subperíodos analisados - tiveram impacto destacado nos indicadores das bases internacionais. O contrário igualmente se verificou, reforçando a evidência pretendida.

Pesquisadores da área de Geociências, cujas pesquisas são usualmente centradas em estudos de solo, datação geológica de regiões, hidrologia, dentre outras, afirmam que seus resultados tendem a ter uma abrangência localizada. Quando ouvidos em estudo anterior (SANTANA, 2018), percebe-se que as estratégias de publicação poderão resultar em duas realidades: estudos de impacto ambiental e social que resultam em mudança ou criação de políticas públicas de alcance local ou nacional; estudos de análise de métodos e tecnologias que visam prover os profissionais da área com inovação e pesquisas de ponta. Tais fatores contribuem para que os pesquisadores da área optem por publicar em periódicos domésticos, viabilizando a aplicação do conhecimento gerado.

Não se pretendeu com esta pesquisa analisar os critérios de seleção de cada base de dados, mas sim verificar o nível de cobertura expresso pela coleção disponível no momento da recuperação da produção. Dessa forma, a Scopus se mostrou mais representativa em números absolutos de documentos indexados e 


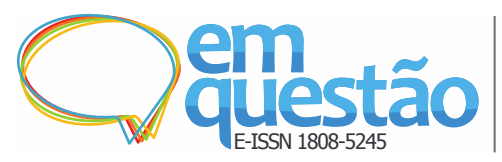

\section{A internacionalização nas Geociências da USP: comparação entre coberturas da Web of Science e da Scopus no nível micro \\ Anderson de Santana e Rogério Mugnaini}

citações recebidas. Contudo, ao se considerar medidas relativas, a vantagem da Scopus não se pronunciou na mesma magnitude, ainda que tenha sobressaído.

Sugerimos a continuação deste estudo, a partir de um detalhamamento do conjunto de periódicos envolvidos, pelo fato de haverem aqueles específicos de cada base, e um maior percentual comum.

\section{Referências}

BALBACHEVSKY, Elizabeth. A pós-graduação no brasil novos desafios para uma política bem-sucedida. In: SCHWARTZMAN, S.; BROCK, C. (Ed.). Os desafios da Educação no Brasil. Rio de Janeiro: Nova Fronteira, 2005. p. 275304.

BUELA-CASAL, Gualberto.; PERAKAKIS, Pandelis.; TAYLOR, Michael.; CHECA, Purificación. Measuring internationality: Reflections and perspectives on academic journals. Scientometrics, v. 67, n. 1, p. 45-65, 2006.

http://doi.org/10.1556/Scient.67.2006.1.4

CAPES - COORDENAÇÃO DE APERFEIÇOAMENTO DE PESSOAL DE NÍVEL SUPERIOR. Documento de Área: Geociências. Brasília, DF: CAPES, 2016. Disponível em:

http://www.capes.gov.br/images/documentos/Documentos_de_area_2017/05_G EOC_docarea_2016.pdf. Acesso em: 17 maio 2018.

COSTAS, Rodrigo.; VAN LEEUWEN, Thed N.; BORDONS, María. A bibliometric classificatory approach for the study and assessment of research performance at the individual level: The effects of age on productivity and impact. Journal of the American Society for Information Science and Technology, v. 61, n. 8, p. 1564-1581, 2010. https://doi.org/10.1002/asi.21348

GLÄNZEL, Wolfgang.; DEBACKERE, Koenraad. On the opportunities and limitations in using bibliometric indicators in a policy relevant context. In: BALL, Rafael. (Ed.). Bibliometric analysis in science and research: applications, benefits and limitations (2nd Conference of the Central Library, Jülich 5.- 7. November 2003 - conference proceedings). [s.1.] Forschungszentrum Jülich, 2003. p. 225-236.

GORRAIZ, Juan.; WIELAND, Martin.; GUMPENBERGER, Christian. Individual bibliometric assessment at University of Vienna: From numbers to multidimensional profiles. El Profesional de la Información, v. 25, n. 6, p. 901, 14 nov. 2016. https://doi.org/10.3145/epi.2016.nov.07

HAUSTEIN, Stefanie.; LARIVIÈRE, Vincent. The Use of Bibliometrics for Assessing Research: Possibilities, Limitations and Adverse Effects. In: WELPE, Isabell M.; WOLLERSHEIM, Jutta; RINGELHAN, Stefanie; Osterloh, Margit E(Ed.). Incentives and Performance. Cham, Switzerland: Springer International Publishing, 2015. p. 121-139. 
LEITE, Paula.; MUGNAINI, Rogério.; LETA, Jacqueline. A new indicator for international visibility: exploring Brazilian scientific community.

Scientometrics, v. 88, n. 1, p. 311-319, 9 jul. 2011.

http://doi.org/10.1007/s11192-011-0379-9

MARRARA, Thiago. Internacionalização da Pós-Graduação: objetivos, formas e avaliação. Revista Brasileira de Pós-Graduação, v. 4, n. 8, p. 245-262, 2007. https://doi.org/10.21713/2358-2332.2007.v4.132

MUGNAINI, Rogério.; DIGIAMPETRI, Luciano. Antonio. The Brazilian national impact: movement of journals between Bradford Zones of production and consumption. In: INTERNATIONAL CONFERENCE ON

SCIENTOMETRICS AND INFORMETRICS, 15, Istambul. Anais... Istambul: Bogaziçi University Printhouse, 2015.

MUGNAINI, Rogério.; DIGIAMPETRI, Luciano. Antonio.; MENA-CHALCO, Jesús. Pascual. Comunicação científica no Brasil (1998-2012): indexação, crescimento, fluxo e dispersão. Transinformação, v. 26, n. 3, p. 239-252, 2014. http://doi.org/10.1590/0103-37862014000300002

OLIVEIRA, Pedro. Vitoriano.; AGOPYAN, Vahan. Avaliação Institucional na USP. In: MUGNAINI, Rogério.; FUJINO, Asa.; KOBASHI, Nair. Yumiko. (Ed.). Bibliometria e cientometria no Brasil: infraestrutura para avaliação da pesquisa científica na era do Big Data. São Paulo: Universidade de São Paulo. Escola de Comunicações e Artes, 2017. p. 111-132. Disponível em: http://www.livrosabertos.sibi.usp.br/portaldelivrosUSP/catalog/book/129. Acesso em: 17 maio 2018.

OLIVEIRA, Talita. Moreira. de; AMARAL, Livio. Políticas Públicas em Ciência e Tecnologia no Brasil: desafios e propostas para utilização de indicadores na avaliação. In: MUGNAINI, Rogério.; FUJINO, Asa.; KOBASHI, Nair. Yumiko. (Ed.). Bibliometria e cientometria no Brasil: infraestrutura para avaliação da pesquisa científica na era do Big Data. São Paulo: Universidade de São Paulo. Escola de Comunicações e Artes, 2017. p. 157-188. Disponível em: http://www.livrosabertos.sibi.usp.br/portaldelivrosUSP/catalog/book/129. Acesso em: 17 maio 2018.

RÀFOLS, Ismael.; MOLAS-GALLART, Jordi.; CHAVARRO, Diego. Andres.; ROBINSON-GARCÍA, Nicolás. On the Dominance of Quantitative Evaluation in Peripherall Countries: Auditing Research with Technologies of Distance.

SSRN, January 2016. Disponível em: http://www.ssrn.com/abstract=2818335. Acesso em: 3 jun. 2017.

SANTANA, Anderson. Avaliação bibliométrica individual:

internacionalização e perfis contextualizados nas Geociências. 2018. 291 f.

Dissertação (Mestrado) - Programa de Pós-Graduação em Ciência da Informação, Escola de Comunicações e Artes, Universidade de São Paulo, São Paulo, 2018. Disponível em:

http://www.teses.usp.br/teses/disponiveis/27/27163/tde-30112018-181842/. Acesso em: 21 jan. 2019. 
SANTANA, Anderson. ; MUGNAINI, Rogério. Internacionalização e impacto nas Geociências: estudo sobre a performance individual de pesquisadores. In: ENCONTRO BRASILEIRO DE BIBLIOMETRIA E CIENTOMETRIA, 6., 2018, Rio de Janeiro. Anais... Rio de Janeiro: UFRJ, 2018. p. 281-289.

ZITT, Michel.; BASSECOULARD, Elise. Internationalization of scientific journals: a measurement based on publication and citation scope.

Scientometrics, v. 41, n. 1-2, p. 255-271, 1998.

http://doi.org/10.1007/BF02457982

\title{
The internationalization in the Geosciences of USP: comparison between Web of Science and Scopus coverage at the micro level
}

\begin{abstract}
The study aimed to analyze the internationalization of the scientific production of the Institute of Geosciences of University of São Paulo (USP) through the application of a set of bibliometric indicators. It is a case study consisting of data collection and bibliometric analysis based on an exploratory and descriptive approach. A sample of 18 researchers was defined, through a set of three categories of indicators: production (Dedalus/USP, Web of Science and Scopus), impact and collaboration (only based on international databases). The results showed that the majority of the researchers of the sample increased their relative number of international publications. In addition, their production was more representative in Scopus, when compared through indicators based on absolute numbers of indexed documents and citations received. However, when considering normalized indicators, Scopus's advantage was not pronounced in the same magnitude. Finally, we cannot ignore the specificities of the areas, which are evident in the practices of scientific communication, which includes several factors of choice of scientific journals, consequently implying the internationalization.
\end{abstract}

Keywords: Internationalization. Bibliometrics. Geosciences. University of São Paulo. Individual Performance.

Recebido: 06/09/2018 Aceito: 03/12/2018

\footnotetext{
${ }^{1}$ Dados da WoS (<webofknowledge.com>) - comercializada pela Clarivate Analytics - e da Scopus (<scopus.com>) - Elsevier -, consultados no dia 10 de setembro de 2018.

${ }^{2}$ Informações sobre Bolsas do CNPq: 〈http://cnpq.br/apresentacao13/>. Acesso em: 10. set. 2018-

3 Journal Citation Reports (JCR). É um produto da WoS, sendo comercializado pela mesma empresa, Clarivate Analytics. Disponível em : <http://jcr.incites.thomsonreuters.com>. Acesso em: 10. set. 2018.

${ }^{4}$ SCImago Journal Ranking (SJR). Disponível em: <https://www.scimagojr.com>. Acesso em: 10. set. 2018:

${ }^{5}$ Consulta aos sítios InCites (<https://incites.thomsonreuters.com〉) e SciVal (<https://scival.com>), no dia 10 de setembro de 2018.
} 